\title{
Decay of Electric Charge on Corona Discharge Polyethylene
}

\author{
L. ZHANG ${ }^{a}$, Z. XU ${ }^{b}$ AND G. $\mathrm{CHEN}^{b}$ \\ ${ }^{a}$ Institute of Fluid Physics, CAEP, P.O. Box 919-150, Mianyang 621900, China \\ ${ }^{b}$ School of Electrical and Computer Science, Southampton University, UK
}

\begin{abstract}
In this paper, surface potential decay after corona charge deposition has been investigated for low density polyethylene (LDPE) films. We believe that the three ways of electron charge decay on the surface of insulating polyethylene film is reasonable. The factor of neutralizing with opposite charge in air is negligible, and leakage along insulator surface or through the body is much more important for electron charge decay. Both ways are related with surface voltage of the sample. When the voltage is lower than a critical value, surface leakage contributes most. If the voltage is higher than this value, the electron energy on the surface of the sample exceeds the critical value and it is easy to cross the "deep trap". In this situation, the body leakage is more important, and the decay on the surface of sample speeds. That is why there exists decay curve crossing phenomenon.
\end{abstract}

PACS numbers: 52.80.-s, 81.05.Lg, 84.30.Jc

\section{Introduction}

It is not completely understood how electric charges on insulation material surface decays. Generally, there are three possible ways, i.e. is neutralized by charges in air, is released on material surface and released through the insulator itself. In the first part of this paper, we studied the decay of surface electric charge on low mass density polyethylene film, which was charged by traditional corona discharge. Compact JCI140 solid detector was used to measure the surface voltage change after the polyethylene was charged.

Many papers had reported the decay of surface electric charge on corona charge polyethylene, but the physical mechanism of decaying process have not been fully understood $[1,2]$. One theory believes that there is a "deep trap" on the surface, while a "shallow trap" in the material. When corona discharge charges the polyethylene, the surface of the film is at a high potential and electrons travel across the "deep trap" much more easily due to higher energy, therefore electric charge on the surface decays faster. If the film is less charged by corona discharge, that the surface is at a low potential, then electron with less total energy cannot travel across the "deep trap" so easily. As a result, the decay of electric charge will be slower. The experimental works in the second part of this paper were used to verify hypothesis of this theory. To make the experimental results more reliable and persuasive, we use pulsed electro-acoustic (PEA) technology to detect charge distribution inside of the insulation material.

\section{Experimental research}

Figure 1 is our experiment setup. Polyethylene (PE) film sample is charged by corona discharge device. A grid electrode was added between discharging electrode and ground to make sure the sample was uniformly charged. The grid was also charged to a fixed potential so that the sample potential was in a fixed range.

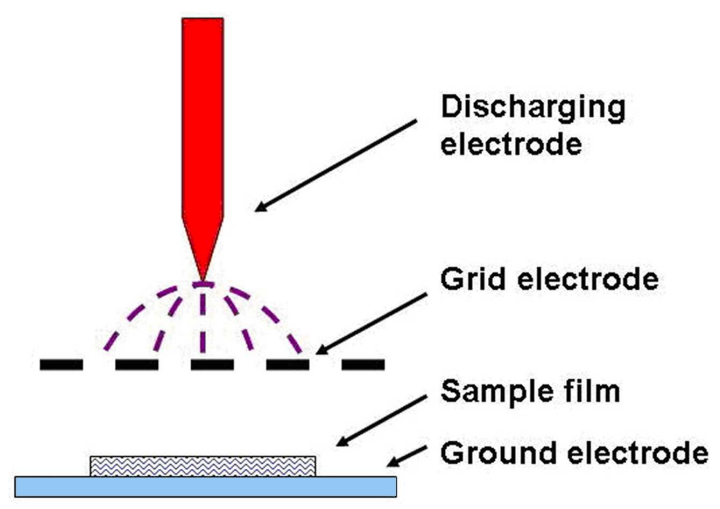

Fig. 1. Experiment setup.

Compact JCI140 solid detector was used to measure the surface potential change of the sample. The sample was charged for $1 \mathrm{~min}$ in the corona charging device. After that, the sample was quickly moved to the JCI150 solid detector to observe the decay of surface voltage, and then decay of surface electric charge can be learned. In order to find the decay law of surface charge, we did the experiment at different electric field by changing potential of both discharging electrode and grid electrode and thickness of the polyethylene film. To facilitate experiment data analysis, we kept the potential fall between discharging and grid electrode at $9 \mathrm{kV}$.

Figure 2 indicates surface potential decay curve at different electrode voltage while keeping potential fall be- 
tween discharging and grid electrode at $9 \mathrm{kV}$, and unchanged thickness of films. From the decay curve, it can be concluded that the higher discharging voltage is, i.e. higher initial electric field on sample, the faster is the voltage decay.

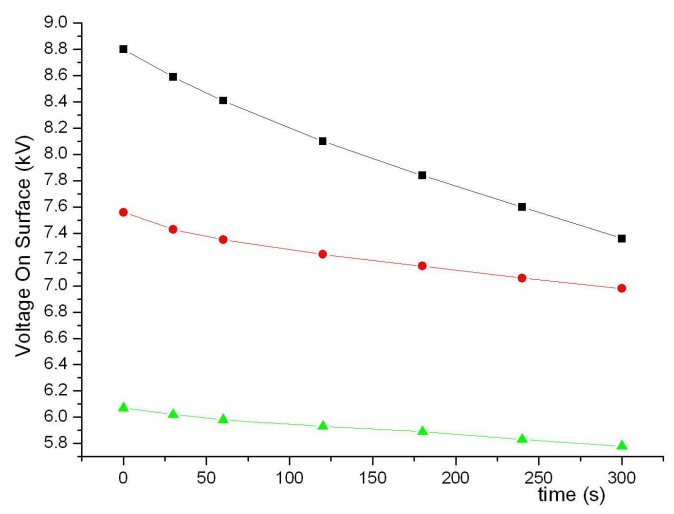

Fig. 2. Surface voltage decay curve at different electrode voltages (squares-highest, triangles-lowest).

Changing the thickness of polyethylene films while keeping charging voltage unchanged, decay curve of surface voltage was derived at different electric fields (see Fig. 3). From the decay curve we can see that higher inner electric fields in sample result in faster voltage decay (it means that electrons travel across the "deep trap" easily at higher electronic field).

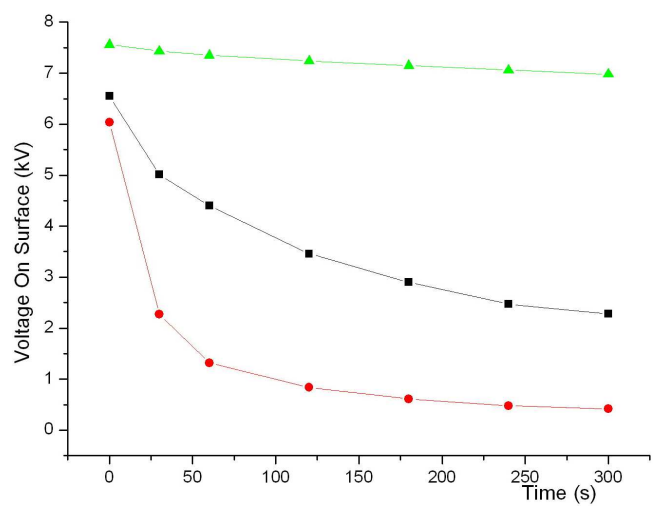

Fig. 3. Surface voltage decay curve at different layers of polyethylene films (see Table II).

In the experiment of changing sample thickness, we chose three samples with thickness of $50 \mu \mathrm{m}, 100 \mu \mathrm{m}$, $180 \mu \mathrm{m}$, respectively. We found that the decay speed is variable with film's thickness and insulation material itself. To eliminate the intrinsic material property influence on experiment result, we did experiment with multi-layer sample of $50 \mu \mathrm{m}$. A three-layer film of $50 \mu \mathrm{m}$ was superposed to keep material property conformable when thickness is increasing. We found that voltage could be measured from the second layer when moved first layer away, and electron were still accumulated on the third layer because voltage could be measured on the third layer. Obviously, electron went through the insulator.

To get further information of electron going through the insulator, we measured charge distribution in a two-layer insulation material sample with PE method. Indeed, we found there were charge distribution (see Fig. 4) inside of the insulator after the sample was charged on the corona discharge setup. The special distribution state at the intersection surface of the two films indicates that there are indeed "deep trap" on the surface and "shallow trap" in the body of the insulator.

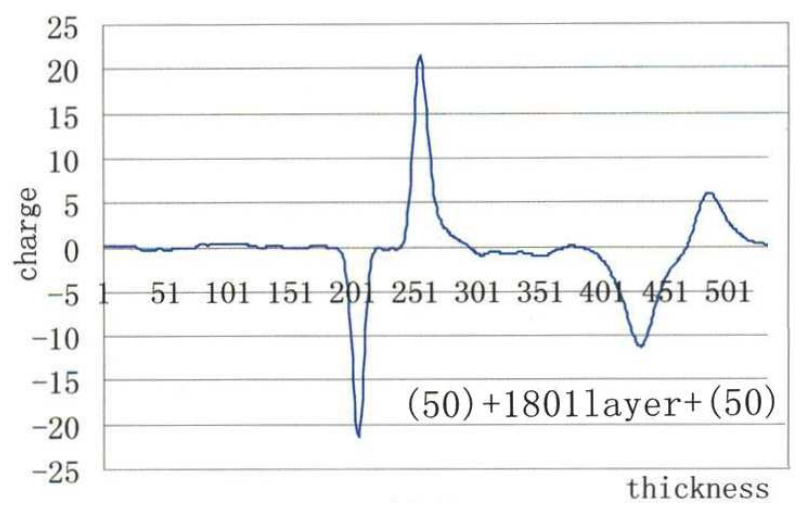

Fig. 4. Charge distribution measured by PE method.

Meanwhile, we found that the data we measured in experiment fluctuated statistically, which is reasonable because samples and their surface status as well as the locus of sample and detector are different in each experiment. Therefore, we statistically average the data to make the result more reliable.

\section{Experimental result and discussion}

Generally, electric charge on the surface of insulation material decays in three ways, i.e. is neutralized by charges in air, flows to ground along material surface or through the insulator itself.

Firstly, we discuss the factor of neutralizing with charges in the air. Charges in air are in low density and move in Brownian movement. Only the charges closed to the sample surface are possible to neutralize with charges on the surface of insulation material. Those charges far from the sample need a long time to reach the sample surface and neutralize the charges on the sample. Although the attractive force to the charge particle in air increases as the sample surface potential increases, the effect is negligible. So, electric charges on insulation material decay mainly by leaking along the surface and through the body.

Therefore, the main way of decay of electric charges on insulating material is leaking over the surface and through the body.

We found that the decay speed did not differ by much at the same charging voltage $(17 \mathrm{kV}$ for discharging elec- 
trode and $8 \mathrm{kV}$ for grid electrode) and different film thickness $(180 \mu \mathrm{m}, 2 \times 180 \mu \mathrm{m}, 3 \times 180 \mu \mathrm{m}, 4 \times 180 \mu \mathrm{m})$. As Fig. 5 shows, Table I marks sample voltages after charging and decaying for $5 \mathrm{~min}$ at different thickness of samples. The voltage of sample was measured in JCI140 detector, and used statistical average.

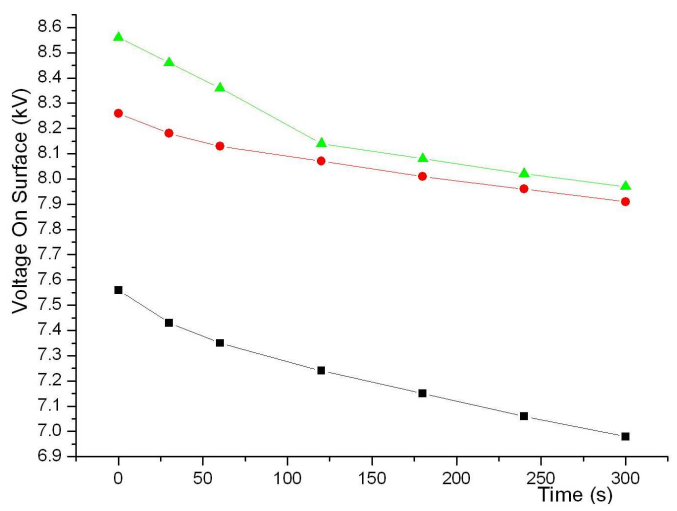

Fig. 5. Surface voltage decay curve at different thickness of polyethylene films (see Table I).

TABLE I

Surface voltage at different thickness.

\begin{tabular}{l|c|c|c}
\hline \hline Thickness $[\mu \mathrm{m}]$ & 180 & $3 \times 180$ & $4 \times 180$ \\
\hline $\begin{array}{c}\text { sample voltage after } \\
\text { charging }\end{array}$ & 7.58 & 8.09 & 8.56 \\
$\begin{array}{c}\text { sample voltage after } \\
\text { decaying for 5 min }\end{array}$ & 7.05 & 7.30 & 7.97
\end{tabular}

The initiative voltage increases as the thickness of film increases. That means the leakage decreases during the 1 min charging process. We consider that the decreasing part is in body leakage, because the conditions of surface leakage have not changed. When the thickness increases, the electric field in the insulation polyethylene film will decrease. So, the probability for electron travelling across the "deep trap" on the surface decreases. The situation is the same for three and four layers. It is a saturation state.

We did the same experiment at the same charging voltage $(17 \mathrm{kV}$ for discharging electrode and $8 \mathrm{kV}$ for grid electrode) and different film thickness $(50 \mu \mathrm{m}, 100 \mu \mathrm{m}$, $180 \mu \mathrm{m})$. The decay curve of surface voltage is shown in Fig. 3; Table II marks sample voltages after charging and decaying for $5 \mathrm{~min}$ at different thickness of samples.
The decay speed is faster as the increase of body electric field in the sample's body. But there is an exceptional result while doing experiment with sample of $100 \mu \mathrm{m}$ and $50 \mu \mathrm{m}$. The sample with thickness of $50 \mu \mathrm{m}$ has a slower decay speed than the sample with thickness of $100 \mu \mathrm{m}$. Maybe it is due to different sample materials.

We also did experiments at different voltage on discharging electrode while fixing the thickness of sample.

$$
\text { TABLE II }
$$

Surface voltage at different thickness.

\begin{tabular}{l|c|c|c}
\hline \hline Thickness $[\mu \mathrm{m}]$ & 50 & 100 & 180 \\
\hline $\begin{array}{c}\text { sample voltage after } \\
\text { charging }\end{array}$ & 6.55 & 6.04 & 7.56 \\
$\begin{array}{c}\text { sample voltage after } \\
\text { decaying for 5 min }\end{array}$ & 2.28 & 0.42 & 6.98
\end{tabular}

We found as the voltage of discharging electrode increases, the decay of the surface voltage speeds up. We conclude that the increase of the sample's voltage makes the accumulated electron energy increase, and it is advantageous for electron to travel across the "deep trap" on the sample surface, which speeds up current leakage through body and then decay of surface electron speeds.

As conclusions for the experiments, we believe that the three ways of electron charge decay on the surface of insulating polyethylene film are reasonable. The factor of neutralizing with opposite charge in air is negligible, and leakage along insulator surface or through the body is much more important for electron charge decay. Both ways are related with surface voltage of the sample. The leakage current is changing with the voltage. When the voltage is lower than a critical value, surface leakage contributes most. If the voltage is higher than this value, the electron energy on the surface of the sample exceeds the critical value and it is easy to cross the "deep trap". In this situation, the body leakage is more important, and the decay on the surface of sample speeds. That is why there exists decay curve crossing phenomenon.

\section{References}

[1] M. Ieda, G. Sawa, U. Shinohara, Japan J. Appl. Phys. 6, 793 (1967).

[2] D.K. Sas-Gupta, IEEE Trans. Electr. Insul. 25, 3 (1990). 